Revue internationale P.M.E.

Économie et gestion de la petite et moyenne entreprise

\title{
INNOVER EN ÎLE-DE-FRANCE : 32 PRIORITÉS POUR LES PME, Rapports et études de la Chambre de commerce et d'industrie de Paris, Paris, La Documentation française, coll. « Développer et entreprendre », 2007, 219 p.
}

\section{André Joyal}

Volume 21, numéro 2, 2008

Vingt ans de recherche francophone en PME et entrepreneuriat

URI : https://id.erudit.org/iderudit/029436ar

DOI : https://doi.org/10.7202/029436ar

Aller au sommaire du numéro

Éditeur(s)

Presses de l'Université du Québec

ISSN

0776-5436 (imprimé)

1918-9699 (numérique)

Découvrir la revue

Citer ce compte rendu

Joyal, A. (2008). Compte rendu de [INNOVER EN ÎLE-DE-FRANCE : 32 PRIORITÉS POUR LES PME, Rapports et études de la Chambre de commerce et d'industrie de Paris, Paris, La Documentation française, coll. « Développer et entreprendre ", 2007, 219 p.] Revue internationale P.M.E., 21(2), 191-193. https://doi.org/10.7202/029436ar 


\section{Comptes rendus}

\section{INNOVER EN ÎLE-DE-FRANCE: 32 PRIORITÉS POUR LES PME Rapports et études de la Chambre de commerce et d'industrie de Paris Paris, La Documentation française, coll. «Développer et entreprendre», 2007, 219 p.}

Malgré le sous-titre, les 32 priorités n'occupent que huit pages à la toute fin d'un ouvrage qui, pour l'essentiel, met en relation l'innovation et la PME avec une application à la région de l'Île-de-France. Malgré l'existence des gisements de croissance importants dans les PME, on signale que la majorité des études en matière d'innovation se rapporte aux grandes entreprises et à la recherche publique. Pour pallier cette situation, la CCIP a établi six domaines d'actions prioritaires:

- l'accompagnement des PME: projets innovants et accès au financement;

- la coopération centre de recherches/PME: diffusion de l'information et transfert de technologie;

- les relations PME/Grands comptes (comprendre grandes entreprises);

- les pôles de compétitivité (un concept que les Québécois tardent à importer alors qu'ils se sont précipités, à une époque, sur celui des districts industriels ou autres systèmes de production locaux);

- la formation dans le domaine du management de l'innovation et de l'entrepreneuriat;

- les PME au cœur de la stratégie régionale de développement de l'innovation.

Les deux rédactrices de ce rapport ont signalé que la France ne fait pas partie des chefs de file européens en matière d'innovation, si l'on prête foi au classement du Tableau de bord européen de l'innovation. Elles n'ont pas osé préciser où se situe l'Hexagone dans un palmarès dominé par la Suède, la Finlande, la Suisse, l'Allemagne et le Danemark. Comme leur étude porte sur la région Île-de-France, il importe de prendre en compte que le poids démographique des PME (entreprises de moins de 500 salariés) qui s'y trouvent 
représente 99,3\% des établissements et $69 \%$ des salariés alors que celles de moins de 50 salariés comptent pour $96,5 \%$ et un peu moins de la moitié de l'ensemble des salariés.

Ce petit livre (format d'un «Que sais-je?»), de présentation fort agréable, contient un grand nombre de fenêtres, soit pour mettre en évidence une citation d'un entrepreneur interrogé (Paroles d'entrepreneurs) soit pour illustrer un cas (Bonnes pratiques). Comme exemple de ces paroles, voici une opinion que ne nierait pas un entrepreneur québécois:

Ce qui est alarmant, c'est de rencontrer des chefs d'entreprise qui ont des idées et n'arrivent pas à mettre en œuvre leur innovation en raison de la complexité administrative, ou de la difficulté à recruter... ou de la frilosité de leur environnement, notamment financier.

On comprend ainsi pourquoi, selon une enquête, 73 \% des PME de Paris ne connaissent pas les dispositifs de soutien aux PME, alors que seulement $4 \%$ d'entre elles avouent bénéficier d'aides publiques.

«Mieux vaut chercher des clients que perdre son temps à chercher des ressources financières publiques » ne craint pas d'affirmer un autre entrepreneur.

Heureusement, qu'il y a les bonnes pratiques comme le révèle l'exemple de Consultencia: une société de services créée en 2003, hébergée dans la pépinière de la CCIP Soleillet, qui se spécialise dans l'édition de solutions en informatique de gestion pour les PME et les grandes sociétés européennes. Reconnue «Jeune entreprise innovante», Consultencia fut lauréate de «Paris entreprendre 2005» en plus de recevoir le prix Master 2007 de la création de l'innovation, de la richesse, du dynamisme et de l'esprit d'entreprise lors du dernier Salon des entrepreneurs (2007). Comme on le voit, Corneille avait raison d'écrire que la valeur n'attend point le nombre des années.

Outre ces vignettes, de nombreux tableaux et schémas accompagnent le texte de façon à bien illustrer les propos. L'un de ces schémas - le processus tourbillonnaire - est emprunté à un «certain» P.-A. Julien qui veut ainsi montrer comment se présentent les interactions et les allers-retours entre les dimensions et l'innovation dans l'entreprise. Une illustration en couleurs fait voir que le processus d'innovation n'a rien de linéaire, ses différentes composantes s'articulant de manière dynamique avec des interactions entre elles. En fait, il est bien précisé que l'innovation au cœur des PME se manifeste par différentes pratiques souvent imbriquées entre elles. Ces pratiques se veulent parfois rien de moins qu'un jeu. Oui, tous les entrepreneurs interrogés ont fait part de leur amusement à travers l'acte de création. Ce dernier, s'il est aussi vu comme un défi culturel, comporte une dimension collective, 
car il suppose l'engagement de tous les métiers et fonctions de l'entreprise de même que la motivation de tous les acteurs. Enfin, l'innovation se caractérise par une démarche transsectorielle, fait-on observer. On y voit, en effet, une recherche de complémentarité à la faveur d'un croisement de filières et de secteurs d'activité. Oui, reconnaissons-le, si pour une PME exporter est difficile, innover l'est tout autant.

C'est pourquoi, lit-on, les PME appellent de leurs vœux l'émergence d'un «relais» ou d'un «point d'entrée» qui serait le premier interlocuteur de confiance en vue d'orienter les dirigeants de PME. Un schéma illustre ce que pourrait être ce relais susceptible d'orienter les PME vers les organismes spécialisés ayant trait, entre autres, à la veille concurrentielle, au transfert de technologies, au financement public et privé, aux incubateurs et autres pépinières et, bien sûr, aux centres de recherche universitaires ou parapublics. Il s'agit ici de la première priorité retenue par les auteures de ce rapport. Au hasard, parmi les 32 qui sont brièvement décrites, je signale les priorités suivantes:

- créer des interfaces entre financiers et entreprises innovantes pour différentes raisons (!);

- augmenter le nombre d'investisseurs type business angels;

- augmenter les contrats d'affaires avec les grands groupes;

- intégrer dans les cursus des chercheurs, ingénieurs, étudiants des formations à l'entrepreneuriat, au management de l'innovation, etc.;

- promouvoir l'expérience des PME qui innovent.

Les informations données tout au long de l'ouvrage sont accompagnées de nombreuses références bibliographiques et d'adresses Internet fort utiles. Comme si cela ne suffisait pas, des annexes offrent au lecteur davantage de bonnes adresses Internet présentées par catégories. Avis donc aux baladeurs du quai Voltaire: un arrêt à La Documentation française vous permettra de glisser cet ouvrage dans votre poche après, bien sûr, être passés à la caisse. Vous en aurez pour votre argent.

André Joyal

Institut de recherche sur les PME, UQTR

Revue internationale P.M.E., vol. 21, n 2, 2008 